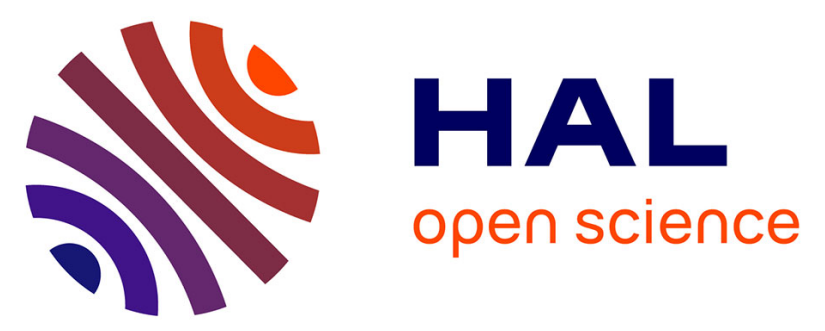

\title{
Pathological findings and prostate-specific antigen outcomes after laparoscopic radical prostatectomy for high-risk prostate cancer.
}

Guillaume Ploussard, Laurent J. Salomon, Yves Allory, Stéphane Terry, Dimitri Vordos, Andreas Hoznek, Claude-Clément Abbou, Francis Vacherot, Alexandre de La Taille

\section{To cite this version:}

Guillaume Ploussard, Laurent J. Salomon, Yves Allory, Stéphane Terry, Dimitri Vordos, et al.. Pathological findings and prostate-specific antigen outcomes after laparoscopic radical prostatectomy for high-risk prostate cancer.. BJU International, 2010, 106 (1), pp.86-90. 10.1111/j.1464410X.2009.09080.x . inserm-00502462

HAL Id: inserm-00502462 https://www.hal.inserm.fr/inserm-00502462

Submitted on 22 Nov 2010

HAL is a multi-disciplinary open access archive for the deposit and dissemination of scientific research documents, whether they are published or not. The documents may come from teaching and research institutions in France or abroad, or from public or private research centers.
L'archive ouverte pluridisciplinaire HAL, est destinée au dépôt et à la diffusion de documents scientifiques de niveau recherche, publiés ou non, émanant des établissements d'enseignement et de recherche français ou étrangers, des laboratoires publics ou privés. 
Guillaume Ploussard, Laurent Salomon, Yves Allory, Stéphane Terry, Dimitri Vordos, Andreas Hoznek,

Correspondence:

Dr A. de la Taille

INSERM U955 Eq07 Department of Urology

CHU Mondor

51, avenue du Maréchal de Lattre de Tassigny

94000 Créteil, France

Tel: 33149812254

Fax: 33149812568

Email: adelataille@hotmail.com

Keywords : prostate cancer ; high risk ; radical prostatectomy ; biochemical recurrence

CHU Henri Mondor, Créteil, France 
Objective: The aim of our study was to review the biochemical recurrence-free survival (RFS) rates of laparoscopic radical prostatectomy (RP) in patients with high risk of disease progression as defined by preoperative d'Amico criteria.

Patients and Methods: Between October 2000 and May 2008, 110 patients underwent extraperitoneal laparoscopic radical prostatectomy and bilateral pelvic lymph node sampling for high risk prostate cancer in our department. High-risk prostate cancer was defined as follows: a PSA level greater than $20 \mathrm{ng} / \mathrm{ml}$, and/or a biopsy Gleason score 8 or above, and/or a clinical AJCC T2c to T4 stage. Median follow-up was 37.6 months. Risk factors for time to biochemical recurrence were tested using log rank survivorship analysis and Cox proportional hazards regression.

Results: Prostate cancer was organ-confined in $35.5 \%$ of cases. Overall RFS was $79.4 \%$ and $69.8 \%$ at 1 and 3 years, respectively. The 3-year RFS rates for organconfined cancer versus extracapsular extension were $100 \%$ and $54.3 \%$, respectively $(p<0.001)$. The 3-year RFS rates for tumor-free seminal vesicle versus seminal vesicle invasion were $81.8 \%$ and $33.6 \%$, respectively $(\mathrm{p}<0.001)$. The 3 -year RFS rates for negative surgical margins versus positive were $85.2 \%$ and $47.3 \%$, respectively $(\mathrm{p}=0.001)$. Compared with men with any single pathological risk factor or any 2 risk factors, men with all 3 risk factors had significantly shorter time to PSA failure after RP (log-rank test: $\mathrm{p}<0.001)$. 

preoperative d'Amico criteria, a third of men with organ-confined disease have favorable prognosis. Men at high risk for early PSA failure could be better identified by pathological assessment on prostatectomy specimens, and selected for phase III randomized trials investigating adjuvant systemic treatment. 
Prostate cancer is the most common solid malignancy in men in EU, with 186,320 new cases diagnosed each year and the second cause of death attributable to cancer with 28,660 deaths per year [1]. Despite the widespread use of prostate-specific antigen (PSA) screening some patients are diagnosed with a locally advanced prostate cancer. In these cases, treatment options remains unclear with no clear consensus. D'Amico et al proposed a useful classification using clinical and pathological parameters to classify relapse risk before treatment [2]. Patients with a PSA > $20 \mathrm{ng}$ / $\mathrm{mL}$, Gleason 8-10, T2c to T4 disease are considered to be at high risk, with recurrence rates ranged from 50 to 100 percent after a local therapy alone especially if they are young, healthy and with a long life expectancy. A nomogram from the Memorial Sloan-Kettering Center has also been validated to predict biochemical recurrence-free survival (RFS) after radical prostatectomy (RP) [3]. For patients with organ-confined and high risk of disease progression prostate cancer, external beam radiation therapy and RP are two recommended treatment options. One important advantage for RP is that cancer aggressiveness is correctly evaluated on RP specimen. Thus, postoperative nomograms can be used to better characterize high risk patients and predict the probability of prostate cancer recurrence for each patient [4]. Pathological risk factors for disease recurrence and disease specific survival after radical prostatectomy (RP) include extracapsular extension, high Gleason score, positive surgical margins, seminal vesicle invasion and positive lymph nodes [5]. Despite treatment, a significant proportion of these patients will experience PSA-defined failure and cancer-specific death indicating a need for more aggressive initial therapy. However, no adjuvant standard treatment after surgery is clearly recommended for high risk and locally 
advanced tumors. Immediate adjuvant radiotherapy decreased the risk of PSA recurrence but at the cost of increased toxicity and with no metastasis-free or overall survival benefit $[6,7]$. Adjuvant hormone therapy significantly improves survival in patients with positive lymph nodes with benefit for immediate therapy [8,9]. In case of negative lymph nodes, this survival advantage is not demonstrated. Recently, neoadjuvant or adjuvant therapy for patients with high risk prostate cancer was studied and demonstrated feasible with acceptable toxicity $[10,11,12]$. Therefore, stratifying patients according to the prognosis is important for postoperative counseling and the consideration of adjuvant therapy. significantly associated with the time to PSA failure.

96 
Study population.

Between October 2000 and May 2008, 110 patients underwent extraperitoneal laparoscopic radical prostatectomy and bilateral pelvic lymph node sampling for high risk prostate cancer in our department. High risk cancers were defined as follows: a PSA level greater than $20 \mathrm{ng} / \mathrm{mL}$, and/or a Gleason score of $\geq 8-10$, and/or clinical $\mathrm{T} 2 \mathrm{c}$ to $\mathrm{T} 4$ disease regarding d'Amico criteria [2]. The patients have negative bone scan and negative computed tomography (CT) scan. Baseline and follow-up information were collected prospectively in our database including preoperative clinical and biological characteristics, patient demographics, surgical data and postoperative parameters. The clinical stage was determined from the digital rectal examination findings according to the AJCC staging system. A standard pelvic lymph node dissection (external iliac artery area) was performed in all patients. A mean of 7.4 lymph nodes were sampled. Nerve-sparing surgery was performed in 34 patients (30.9\%). Pathological Gleason score, surgical margin (SM) status, presence of extracapsular extension (ECE), seminal vesicle invasion (SVI) and pelvic lymph node positivity were recorded. All prostatectomy specimens were assessed by a referee genitourinary pathologist. Biochemical recurrence was defined as any detectable serum PSA (greater than $0.2 \mathrm{ng} / \mathrm{ml}$ ). Patients who had pelvic lymph nodes metastases received immediate androgen deprivation therapy. Others patients who received adjuvant or neoadjuvant hormonal and/or radiation therapy before PSA failure which was the primary end point in this study, were excluded from analyses. Men who experienced PSA failure were managed according to standard practice. Table 1 lists the preoperative characteristics of the 110 patients. Patients were classified at higher 
risk of disease progression on PSA level alone in 61 patients (55.5\%), on high Gleason score alone in 31 patients $(28.2 \%)$, on combination of these two parameters in 9 patients $(8.2 \%)$ and on clinical AJCC stage alone in 9 patients $(8.2 \%)$. The mean and median follow-up for all patients was 38.1 and 37.6 months, respectively (range: 1.1-92.2). The frequency of follow-up visits was standardized for all patients.

Statistical analysis.

Preoperative clinico-biological parameters were studied according to time-to-PSA failure. We used dichotomized values of preoperative PSA (20 or less versus greater than $20 \mathrm{ng} / \mathrm{ml}$ ), prostate weight (30 or less versus greater than $30 \mathrm{ml})$ and age $(<60$ versus $>60$ years old). A Cox proportional hazards regression was used. For purpose of illustration, estimates of PSA outcomes were calculated using the Kaplan-Meier actuarial method and graphically displayed. PSA failure was defined by a PSA level $>0.2 \mathrm{mg} / \mathrm{ml}$. The time to PSA failure was considered to be the time that the first detectable value was measured. The day of surgery was reported as the starting point of analysis. The analysis endpoint was biochemical recurrence-free survival (RFS). Postoperative significant risk factors for time to biochemical recurrence were examined using log rank survivorship analysis. All data were analyzed using SPSS 13.0 software (Chicago, Illinois). The limit of statistical significance was defined as $\mathrm{p}<0.05$. 
Pathological parameters on prostatectomy specimens (see Table2)

Of the 110 patients undergoing RP, the pathological findings were extracapsular extension (ECE) in 71 (64.5\%), seminal vesicle involvement (SVI) in 27 (24.5\%), positive surgical margin $(\mathrm{R}+)$ in $43(39.1 \%)$ and pelvic lymph node metastases $(\mathrm{N}+)$ in $4(3.6 \%)$. Surgical margins were positive in $21.0 \%, 27.3 \%, 61.1 \%$ and $71.4 \%$ of pT2, pT3a, pT3b and pT4 cancers, respectively.

Concordance between biopsy and postprostatectomy Gleason scores was $49.1 \%$. Biopsy upgraded pathological Gleason score in 16 patients (14.5\%) and downgraded it in 40 patients (36.4\%). The rate of favourable disease (defined as pT2 cancer and a Gleason score $\leq 7$ ) on RP specimens was $26.4 \%$. Only 7 patients (6.4\%) fulfilled all favourable criteria, i.e. a PSA $<20 \mathrm{mg} / \mathrm{ml}$, a pT2 cancer and a Gleason score $\leq 7$ on RP specimen.

\section{Time to PSA failure analyses}

During a mean follow-up of 38.1 months, PSA failure occurred in 23 patients (20.9\%) of which 20 received salvage therapy (radiotherapy or hormonal therapy). Fourteen patients were treated by radiotherapy and 6 men received androgen deprivation therapy. Mean time to progression was 7.2 months. No patient died as a result of prostate cancer before PSA failure.

Multivariate time-to-failure analysis on preoperative parameters showed PSA level higher than $20 \mathrm{ng} / \mathrm{mL}(\mathrm{p}=0.003 ; \mathrm{HR}=7.14$ [95\% CI 1.93-26.3]) and high biopsy Gleason score $(\mathrm{p}=0.023 ; \mathrm{HR}=3.14$ [95\% CI 1.17-8.43]) to be independent predictors 
of biochemical recurrence. Age ( $\mathrm{p}=0.658 ; \mathrm{HR}=0.82$ [95\% CI 0.34-1.95]), clinical stage $(\mathrm{p}=0.362 ; \mathrm{HR}=1.49$ [95\% CI 0.63-3.48]) and prostate weight $(\mathrm{p}=0.530$; $\mathrm{HR}=0.62$ [95\% CI 0.14-2.75]) were not significantly associated with biochemical relapse.

Overall RFS was $79.4 \%$ at 1 year (95\% confidence interval [CI]: 75.1-83.7\%) and $69.8 \%$ at 3 years (95\% CI 63.9-75.7\%) (Figure 1).

\section{PSA failure stratified by pathological data (Figure 2)}

The 3-year RFS rates for organ-confined cancer versus extracapsular extension were $100 \%$ and $54.3 \%$ (95\% CI 46.3-62.3\%), respectively ( $\mathrm{p}<0.001)$. The 3-year RFS rates for tumor-free seminal vesicle versus seminal vesicle invasion were $81.8 \%$ (95\% CI 76.1-87.5\%) and 33.6\% (95\% CI 20.4-46.8\%), respectively ( $<<0.001)$. The 3-year RFS rates for negative surgical margins versus R+ were 85.2\% (95\% CI 79.4-91.0\%) and $47.3 \%$ (95\% CI 36.9-57.7\%), respectively ( $\mathrm{p}=0.001)$.

The estimated rates of RFS stratified by the number of pathological risk factors present are illustrated in Figure 3. When these 3 pathological factors were associated (SVI, R+, ECE), the 1-year and 3-year RFS rates were 42.2\% (95\% CI 29.5-54.9\%) and $15.8 \%$ (95\% CI 2.8-28.8\%). Statistically significant difference appeared according to the number of these factors present (log-rank test: $\mathrm{p}<0.001)$. None of the 9 patients with pT2 cancer and positive margin developed PSA recurrence. Comparatively, the 1-year and 3-year RFS rates were $84 \%$ and $74.7 \%$ in patients with pT3 cancer and negative margin. Difference did not reach significance (log-rank test: $\mathrm{p}=0.185)$. 
An accurate prediction of probability of disease recurrence is essential for proper patient selection. Preoperatively, the identification of high risk prostate cancer can be based on, at least, three well-defined predictors of the disease extent and outcome after treatment: AJCC clinical T stage, serum PSA level, and Gleason score. Patients with AJCC clinical stage T2c-T4 disease and/or a PSA level of more than 20ng/mL and/or a biopsy Gleason score of 8 or more have a risk higher than $50 \%$ at 5 years of post-treatment PSA failure. This risk group was established from a review of literature and well defined by d'Amico et al. studying PSA failure and prostate cancer-specific mortality [2,13]. In addition, PSA velocity greater than $>2 \mathrm{ng} / \mathrm{mL} /$ year and more than $50 \%$ of positive biopsies can be considered $[14,15]$. D'Amico et al reported in a study including 1,095 patients who underwent RP and who did not receive adjuvant therapy that on multivariable analysis, preoperative PSA velocity $>2 \mathrm{ng} / \mathrm{mL} /$ year was associated with an increased risk of cancer specific mortality and with an increased risk of overall mortality [16]. Others preoperative nomograms or scores have been developed and validated in internal and external studies, documenting a high level of consistency $[3,17]$. However, despite a good predictive accuracy among different risk groups, certain inconsistencies have been reported regarding high risk cases. In the present series we studied PSA-defined follow-up and results of local therapy for patients at high risk of cancer progression regarding preoperative d'Amico criteria [2]. Survival analyses were driven in order to identify different risk subgroups of PSA failure according to final pathological assessment among patients suspicious for high risk of biochemical failure. 
In multivariate time-to-failure analysis, high biopsy Gleason score and serum PSA were significantly associated with biochemical relapse in our cohort. Preoperative PSA greater than $20 \mathrm{ng} / \mathrm{ml}$ and a Gleason score 8 or above carried a 7.14 and 3.14-time increased risk of recurrence, respectively. No other preoperative variables were significantly associated with time to PSA failure. However, we did not study PSA velocity in this series [16]. Interestingly, clinical AJCC stage did not appear as significant predictor of biochemical recurrence in this subgroup of high-risk prostate cancer, on the contrary of published data concerning low-risk and intermediate-risk group $[2,13]$. Kupelian et al have already shown that clinical stage was not independent predictor of PSA-defined failure in a population of patients with biopsy Gleason score 8 or above [18]. Our data confirmed these results.

Biochemical control rates were encouraging for these high risk patients. A third of these patients have prolonged disease-free survival. Among the 110 patients, prostate cancer was organ-confined in $35.5 \%$ of cases on final pathological assessment. Biochemical RFS was excellent for this subgroup with no cases of recurrence after a mean follow-up of 37.1 months $( \pm 23.2)$. No early PSA failure appeared in case of organ-confined disease. Moreover, no patient with pT2 cancer and positive surgical margin had PSA failure. Thus, the biology of organ-confined disease appeared different compared with the behavior of pT3 cancers. In this series of high-risk PCa, patients with pT3 cancers and negative surgical margins had poorer survival than those with pT2 cancer and positive margin $(74.7 \%$ versus $100 \%$ at 3 years after the surgery). However, difference failed to show significance. In case of biochemical recurrence, patients with positive surgical margins were preferentially treated by salvage radiotherapy, whereas patients with non organ- 
confined disease or early PSA failure received preferentially androgen deprivation therapy.

As expected, seminal vesicle invasion and positive surgical margins were statistically

strong predictors of early biochemical recurrence [19]. The 3-year RFS rates for tumor-free seminal vesicle versus seminal vesicle invasion were $81.8 \%$ and $33.6 \%$, respectively $(\mathrm{p}<0.001)$. The 3-year RFS rates for negative surgical margins versus $\mathrm{R}+$ were $85.2 \%$ (95\% CI 79.4-91.0\%) and 47.3\% (95\% CI 36.9-57.7\%), respectively $(\mathrm{p}=0.001)$. The biochemical recurrence in patients with 3 combined pathological adverse factors was extremely frequent and early with a 3-year RFS of $15.8 \%$. Therefore, men at high risk for early PSA failure could be identified on the basis this pathological assessment. Not all patients with ECE, positive surgical margins or VSI will fail biochemically postoperatively, but combination of these 3 parameters was reported to be a strong predictor of early PSA failure. Compared with men with any single pathological risk factor or any 2 risk factors, men with all 3 risk factors had significantly shorter time to PSA failure after RP (log-rank test: $\mathrm{p}<0.001)$. However, the overall risk of relapse may be underestimated in spite of the relative short followup of our cohort. The PCa progresses slowly and a median follow-up of 3 years may be considered as insufficient.

Despite the independent statistical significance of two preoperative clinico-biological parameters (PSA level and high Gleason score) to predict time to postoperative PSA failure, most of the variation in PSA follow-up was not accounted for on the basis of the d'Amico criteria. For patients with organ-confined disease, the outcomes were remarkably good with RP. Pathological criteria explained a significant amount of the variation in the postoperative PSA data. Longer follow-up including more patients in 
the higher risk categories would provide stronger conclusions. One reason of the good cure rates might be that PSA failure was defined by a PSA level over $0.2 \mathrm{mg} / \mathrm{ml}$ whereas in other series a " 0.2 or greater" or a " 0.1 " cut-off was used [20]. The use of such a cut-off might be too high when a 3-year RFS is studied and might have limited detection. We'd also like to emphasize that the biochemical recurrence but not the prostate cancer specific mortality has been chosen as criteria of disease progression. The ideal end point on which to make treatment decisions is survival and PSA-defined failure may not accurately reflect the likehood of prostate cancer-specific death. However, early PSA failure is established to be associated with an increased risk of progression to metastatic disease and prostate cancer-specific death [21]. If longer follow-up and rates of specific mortality confirm these results, adjuvant therapy might not be mandatory for achieving adequate cure rates for the subgroup of patients with organ-confined disease. On the contrary, men with two or three pathological risk factors should be considered for phase III randomized trials investigating adjuvant systemic treatment. Integrate taxane-based chemotherapy with local treatment could be relevant to address microscopic hormone-refractory prostate cancer cells that may be present at initial assessment in men with high-risk disease $[10,11,12]$

One limitation of our study is the bias due to the selection of men candidates for RP. During the study period, 97 others patients have been diagnosed with localized PCa at high risk of recurrence according to the d'Amico criteria. These patients have been treated by radiotherapy in 40 cases and by androgen deprivation therapy in 57 elderly patients. We excluded 47 patients who have been included into a prospective trial investigating the role of adjuvant paclitaxel after RP. Adjuvant radiotherapy is not 
performed for high risk PCa before PSA failure at our department and no patient has received neoadjuvant therapy during the study period.

Our findings supported evidence that organ-confined disease, even in preoperatively high risk patients can lead to excellent RFS outcomes after RP without adjuvant therapy. Local therapy such as RP has to remain a standard of care for these high risk patients. Pathological evaluation on prostatectomy specimens provides better predictive assessment of high risk compared with only preoperative criteria. Despite high accuracy and mandatory use for selecting men for clinical trials, high risk group established according to the d'Amico criteria remains heterogeneous, including a third of patients with excellent prognosis. Radical prostatectomy can help to distinguish patients who would benefit from adjuvant therapy and close surveillance, from those who could be watched in a more spaced out way.

Finally, this series confirms that laparoscopic approach is a validated treatment of clinically localized but high-risk disease. Oncological results and positive surgical margins rates were in line with values published in open surgery series [22,23]. 
Three years after laparoscopic radical prostatectomy for high risk prostate cancer, biochemical recurrence-free survival is $69.8 \%$. Our findings support evidence that organ-confined disease, even in preoperatively high risk patients can lead to excellent RFS outcomes after RP without adjuvant therapy. Among these patients with high risk disease defined by preoperative data, a third of men with organ-confined disease have favorable prognosis. Thus, men at high risk for early PSA failure could be better identified by pathological assessment on prostatectomy specimens, and selected for phase III randomized trials investigating adjuvant systemic treatment. 
[2] D'Amico AV, Whittington R, Malkowicz SB, Schultz D, Blank K, Broderick GA, et al. Biochemical outcome after radical prostatectomy, external beam radiation therapy, or interstitial radiation therapy for clinically localized prostate cancer. JAMA 1998; 280: 969-974.

[3] Kattan MW, Eastham JA, Stapleton AM, Wheeler TM, Scardino PT. A preoperative nomogram for disease recurrence following radical prostatectomy for prostate cancer. J Natl Cancer Inst. 1998; 90:766-71.

[4] Kattan MW, Wheeler TM and Scardino PT. Postoperative nomogram for disease recurrence after prostatectomy for prostate cancer. J Clin Oncol 1999; 17:1499-1507.

[5] Roehl KA, Han M, Ramos CG, Antenor JA, Catalona WJ. Cancer progression and survival rates following radical retropubic prostatectomy in 3,478 consecutive patients: long-term results. J Urol 2004; 172: 910-914. Adjuvant radiotherapy for pathologically advanced prostate cancer: a randomized clinical trial. JAMA 2006; 296:2329-35. 
[7] Bolla M, van Poppel H, Collette L, van Cangh P, Vekemans K, Da Pozzo L, et al. European Organization for Research and Treatment of Cancer. Postoperative radiotherapy after radical prostatectomy: a randomised controlled trial (EORTC trial 22911). Lancet 2005 13-19; 366:572-8.

[8] Messing E M, Manola J, Sarosdy M, Wilding G, Crawford ED, Trump D.

Immediate hormonal therapy compared with observation after radical prostatectomy and pelvic lymphadenectomy in men with node-positive prostate cancer. N Engl J Med 1999; 341: 1781-1788.

[9] Messing E M, Manola J, Yao J, Kiernan M, Crawford D, Wilding G, et al. Immediate versus deferred androgen deprivation treatment in patients with nodepositive prostate cancer after radical prostatectomy and pelvic lymphadenectomy. Lancet Oncol 2006; 7: 472-479.

[10] Kibel A S, Rosenbaum E, Kattan M W, Picus J, Dreicer R, Klein EA, et al. Adjuvant weekly docetaxel for patients with high risk prostate cancer after radical prostatectomy: a multi-institutional pilot study. J Urol 2007; 177: 1777-1781.

[11] Sonpavde G, Chi KN, Powles T, Sweeney CJ, Hahn N, Hutson TE, et al. Neoadjuvant therapy followed by prostatectomy for clinically localized prostate cancer. Cancer 2007; 110:2628-39. 
[12] Dreicer R, Magi-Galluzzi C, Zhou M, Rothaermel J, Reuther A, Ulchaker J, et al.

Phase II trial of neoadjuvant docetaxel before radical prostatectomy for locally advanced prostate cancer. Urology 2004; 63:1138-42.

[13] Tsai HK, Chen MH, McLeod DG, Carroll PR, Richie JP, D'Amico AV. Cancerspecific mortality after radiation therapy with short-course hormonal therapy or radical prostatectomy in men with localized, intermediate-risk to high-risk prostate cancer. Cancer 2006; 107:2597-603.

[14] Freedland S J, Humphreys E B, Mangold L A, Eisenberger M, Dorey FJ, Walsh $\mathrm{PC}$,

et al. Risk of prostate cancer-specific mortality following biochemical recurrence after radical prostatectomy. JAMA 2005; 294: 433-439.

[15] Ravery V, Chastang C, Toublanc M, Boccon-Gibod L, Delmas V, Boccon-Gibod L. Percentage of cancer on biopsy cores accurately predicts extracapsular extension and biochemical relapse after radical prostatectomy for T1-T2 prostate cancer. Eur Urol 2000; 37: 449-455.

[16] D'Amico A V, Chen M H, Roehl K A, Catalona WJ. Preoperative PSA velocity and the risk of death from prostate cancer after radical prostatectomy. N Engl J Med 2004; 351: 125-135.

[17] Cooperberg MR, Freedland SJ, Pasta DJ, Elkin EP, Presti JC Jr, Amling CL, et al. Multiinstitutional validation of the UCSF cancer of the prostate risk assessment for prediction of recurrence after radical prostatectomy. Cancer 2006; 107:2384-91. 
[18] Kupelian PA, Buchsbaum JC, Elshaikh M, Reddy CA, Zippe C, Klein EA. Factors affecting recurrence rates after prostatectomy or radiotherapy in localized prostate carcinoma patients with biopsy Gleason score 8 or above. Cancer 2002; 95:2302-7.

[19] D'Amico AV, Whittington R, Malkowicz SB, Schultz D, Schnall M, Tomaszewski JE, et al. A multivariate analysis of clinical and pathological factors that predict for prostate specific antigen failure after radical prostatectomy for prostate cancer. J Urol 1995; 154:131-8.

[20] Pavlovich CP, Trock BJ, Sulman A, Wagner AA, Mettee LZ, Su LM. 3-year actuarial biochemical recurrence-free survival following laparoscopic radical prostatectomy: experience from a tertiary referral center in the United States. J Urol 2008;179:917-21

[21] Freedland SJ, Humphreys EB, Mangold LA, Eisenberger M, Partin AW. Time to prostate specific antigen recurrence after radical prostatectomy and risk of prostate cancer specific mortality. J Urol 2006; 176:1404-8.

[22] Catalona WJ, Smith DS. Cancer recurrence and survival rates after anatomic radical retropubic prostatectomy for prostate cancer: intermediate-term results. J Urol $1998 ; 160: 2428-34$. 
[23] Han M, Partin AW, Pound CR, Epstein JI, Walsh PC. Long-term biochemical disease-free and cancer-specific survival following anatomic radical retropubic prostatectomy. The 15-year Johns Hopkins experience. Urol Clin North Am 2001; 28:555-65.

420

421

422 
Table 2. Pathological postprostatectomy parameters.

Figure 1. Biochemical recurrence-free survival after RP for high risk prostate cancer. present. factors (ECE, VSI, R+). 